\title{
Changes in Body Mass Index after Arthroscopic Surgery and Its Effect on Functional Outcomes
}

\author{
Gökhan Polat'1, Hasan Hüseyin Ceylan'2, Ersin Kuyucu ${ }^{3 *}$, Necdet Demir ${ }^{4}$, \\ Gökhan Karademir ${ }^{1}$, Mehmet Erdil ${ }^{3}$ \\ ${ }^{1}$ Department of Orthopedics and Traumatology, Istanbul University, Istanbul, Turkey \\ ${ }^{2}$ Department of Orthopedics and Traumatology, LNB Sultançiftliği Government Hospital, Istanbul, Turkey \\ ${ }^{3}$ Department of Orthopedics and Traumatology, Istanbul Medipol University, Istanbul, Turkey \\ ${ }^{4}$ Department of Orthopedics and Traumatology, Bezmialem University, Istanbul, Turkey \\ Email: "ersinkuyucu@yahoo.com.tr
}

Received 13 April 2016; accepted 21 May 2016; published 24 May 2016

Copyright (C) 2016 by authors and Scientific Research Publishing Inc.

This work is licensed under the Creative Commons Attribution International License (CC BY).

http://creativecommons.org/licenses/by/4.0/

(c) (7) Open Access

\begin{abstract}
Objective: Arthroscopic surgery is one of the most common surgical procedures in orthopedic surgery. Post-surgery weight gain is one of the most important factors that affects the functional outcomes of arthroscopic surgery. This study investigated the effect of changes in the body mass index (BMI) in the period after arthroscopic surgery and the potential impact of these changes on functional outcomes. Methods: One hundred-sixty four patients (knee and hip arthroscopy patients) were evaluated retrospectively. Changes in the BMI and functional scores of the patients between the preoperative period and first, third, and sixth months postoperatively were compared. Results: The mean age of the patients was 36.03 (15 - 64) years, and the mean follow-up was 14.2 months (6 - 29) months. Postoperative increases in the BMI values in the first, third, and sixth months were statistically significant in the three groups of patients (i.e., meniscal pathology, ligament pathology, and intra-articular hip pathology $(p<0.001)$. The correlations of the BMI and functional score changes in the intra-articular hip pathology (hip arthroscopy) $(p=0.564)$, meniscal pathology $(p=0.854)$, and ligament pathology groups $(p=0.908)$ were not statistically significant. Conclusion: Patients may gain weight following arthroscopic surgery of the knee and hip. Orthopedic surgeons should keep this issue in mind and educate patients about how to prevent potential weight gain to ensure it does not adversely affect functional outcomes.
\end{abstract}

\section{Keywords}

Arthroscopy, Body Mass Index, Weight Gain, Arthroscopy

\footnotetext{
${ }^{*}$ Corresponding author.
}

How to cite this paper: Polat, G., Ceylan, H.H., Kuyucu, E., Demir, N., Karademir, G. and Erdil, M. (2016) Changes in Body Mass Index after Arthroscopic Surgery and Its Effect on Functional Outcomes. International Journal of Clinical Medicine, 7, 293-296. http://dx.doi.org/10.4236/ijcm.2016.75031 


\section{Introduction}

Arthroscopic intervention is one of the most common surgical procedures in orthopedic practice [1] [2]. Following arthroscopic surgery, patients may gain weight due to postoperative immobilization. This post-surgery weight gain can affect functional outcomes because obesity increases the complications, morbidities and mortalities after the surgery [1] [2]. In the literature, this affect has been mentioned for the trauma and hip arthroplasty patients and obesity patients had increased vascular, immunologic and pulmonary complications after the surgery [3] [4]. The aim of this study was to evaluate postoperative body mass index (BMI) changes in patients who underwent arthroscopic surgery and the effects of these changes on the functional outcomes of the patients.

\section{Methods}

The study consisted of 594 patients (147 knee, 111 shoulder, 17 hip, 8 ankle, and 3 elbow arthroscopies) who were treated in two different clinics between October 2010 and May 2013. There was no postoperative mobilization restriction in the shoulder arthroscopy group, so they were excluded. The ankle and elbow arthroscopy cases were also excluded due to a low number of cases, as it would not have been possible to analyze them statistically. The final study included 164 knee and hip arthroscopy patients who underwent surgery for meniscal, ligament, and intra-articular hip pathologies.

The patients' preoperative functional joint scores, heights, weights, and BMI values were obtained from their medical records. In the postoperative period, the functional outcomes were objectively scored and recorded. In the functional assessment, the International Knee Documentation Committee (IKDC) score was used for meniscus injuries, the Lysholm knee score was applied for ligament injuries, and the Harris hip score was used for hip joint diseases. Lysholm and IKDC scores are scales that range from 0 to 100 and 80 - 100 score means perfect result, 60 - 79 score means good, 40 - 59 score means moderate and $<40$ score means poor result. In the postoperative first, third, and sixth months, the functional outcomes and BMI changes were measured and recorded.

Statistical evaluation forgroup comparisons was performed with SPSS v16. The data were measured by Chisquare, Tukey's, Bonferroni and ANOVA tests.

\section{Results}

The study consisted of 164 patients (77 meniscal injury group, 70 ligament injury group and 17 hip arthroscopy group) who were underwent arthroscopic surgery.

Of the 164 patients, 96 (58.5\%) were men, and 68 (41.5\%) were women. The mean follow up was 14.2 (6 - 29) months. According to a chi-square test, the sex distribution was not different between the knee and hip patient groups $(p>0.05)$. The mean age of the patients was $36.03(15-64)$ years. The mean age of the patients in the meniscus and ligament injury groups was $40.9(15$ - 64) and $30.5(17$ - 63) years, respectively. The age distribution of the two groups was similar (Tukey's test, $p>0.05$ ). The mean age of the hip arthroscopy patient group was 36.76 (23 - 62) years (Table 1$)$.

Seventy-seven (46.9\%) of 147 knee arthroscopy cases had meniscal injuries, and the remaining 70 (42.6\%) had cruciate ligament injuries. Sixty-four of the 77 patients with a meniscal injury were treated with debridement, and 13 meniscal tears were repaired. In the cruciate ligament injury group, all the cruciate ligaments were reconstructed using hamstring autografts. None of the 17 (10.3\%) hip labrum tears were repaired. All were debrided.

Preoperatively, the mean BMI of the study groups was 25.29. In the postoperative first, third and sixth months, the BMIs of the study groups were 25.59, 25.68, and 25.72, respectively. The postoperative increase in the BMI was statistically significant (repeated ANOVA, $p<0.001$ ).

Table 1. Demographic results of the patients.

\begin{tabular}{lccc}
\hline & Age (year) & Number of patients & BMI changes between preoperative and postoperative \\
\hline Meniscal injury & $40.9(15-64)$ & 77 & $p<0.001$ \\
Ligament injury & $30.5(17-63)$ & 70 & $p<0.001$ \\
Hip arthroscopy & $36.76(23-62)$ & 17 & $p<0.001$ \\
\hline
\end{tabular}


To evaluate the BMI changes in each group, the Bonferroni test was used. The BMI increased significantly between the preoperative and postoperative periods in the meniscus group $(p<0.001)$. However, the BMI changes between the first, third, and sixth postoperative months were not significant $(p>0.05)$.

In the ligament-injured group, the changes in the BMI were significant between the preoperative period and the three postoperative periods $(p<0.001)$. The BMI changes between the first and sixth $(p=0.005)$ and third and sixth $(p=0.011)$ postoperative months were significant $(p=0.04)$. However, the changes in the BMI between the first and sixth months were not significant $(p=0.912)$.

The changes in the BMI in the hip arthroscopy group between the preoperative period and the postoperative periods were significant $(p<0.001)$. The changes in the BMI between the first month and both third and sixth postoperative months were significant $(p=0.003)$.

The correlation between changes in the BMI and the functional outcomes of the arthroscopy patients in the postoperative sixth month were evaluated using a one-way ANOVA test. When the functional outcomes of each group were compared, there was no correlation in any of the groups: hip arthroscopy group, $p=0.564$; meniscus injury group, $p=0.854$; and ligament injury group, $p=0.908$.

In the study, $110(67.07 \%)$ of the 164 patients had a mean postoperative rehabilitation period of 13.7 (min 2max 45) days, and the remaining patients had no rehabilitation. According to the results of Spearman's analysis, there was no correlation between the rehabilitation period and BMI changes in the rehabilitation group ( $p=$ $0.844)$.

With regard to postoperative weight bearing on the operated extremity, there were no postoperative differences in the BMIs of the three groups.

\section{Discussion}

Arthroscopic interventions are common surgical procedures in orthopedic practice [1] [2]. Many factors influence the postoperative functional outcomes of the surgery, but the main factor is immobilization-induced weight gain [3]-[9]. After hip and knee arthroscopy, as in lower extremity surgeries, the patient's body weight can affect weight-bearing joints, healing, and outcomes [10]. In non-obese patients, many factors can trigger postoperative obesity [3]-[7]. The present study included only weight-bearing lower extremity cases (i.e., not shoulder and elbow injuries).

A previous study of the relationship between the BMI and functional outcomes in 1090 partial meniscectomy cases reported a reverse relation between functional outcomes and obesity [7]. In an 11-year follow-up study of 121 patients who had undergone arthroscopic, interventions, obese patients had lower SF-36 and WOMAC scores than non-obese patients [8]. In the present study, the patients' weights increased significantly after the lower extremity arthroscopic interventions. However, the results revealed no correlation between the BMI changes and functional outcomes. Similar results were reported in two recent studies, which found that obesity did not affect the functional outcomes of patients following arthroscopic treatment of anterolateral ankle impingement and hip arthroscopy [11] [12].

Postoperative physiotherapy and rehabilitation are well-known methods to enhance functional outcomes in the postoperative period by aiding the patient's recovery and preventing gaining weight [13]. In the present study, 110 (67.07\%) of the 164 patients had postoperative rehabilitation. The results revealed no correlation between the rehabilitation period and BMI changes in the rehabilitation group.

This study has some limitations. It was a retrospective study, and the sample size was relatively small. Patients with ankle and elbow injuries had to be excluded due to the low number of cases. None of the patients required labrum repair, which might affected mobilization and potentially increased the BMI. Despite not affecting the mobilization abilities of the patients, the present study showed that arthroscopic surgery on weightbearing lower extremities was a risk factor for weight gain.

Knee and hip arthroscopy patients may gain weight following surgery. Patients should be informed preoperatively about this possibility. Diet regulation or postoperative modifications could be used to prevent excess weight gain. Although the limited increase in the BMIs did not affect functional outcomes, weight gain has other undesirable effects on health. These can be prevented by dietary management following surgery.

\section{Conflict of Interest}

All the authors declared that they have no conflict of interest. 


\section{References}

[1] Klimkiewicz, J.J. and Shaffer, B. (2002) Meniscal Surgery 2002 Update: Indications and Techniques for Resection, Repair, Regeneration, and Replacement. Arthroscopy, 18, 14-25. http://dx.doi.org/10.1053/jars.2002.36505

[2] Rosenberg, T.D., Metcalf, R.W. and Gurley, W.D. (1988) Arthroscopic Meniscectomy. Instructional Course Lectures, 37, 203-208.

[3] Haverkamp, D., Klinkenbijl, M.N., Somford, M.P., Albers, G.H. and van der Vis, H.M. (2011) Obesity in Total Hip Arthroplasty: Does It Really Matter? A Meta-Analysis. Acta Orthopaedica, 82, 417-422. http://dx.doi.org/10.3109/17453674.2011.588859

[4] Bochicchio, G.V., Joshi, M., Bochicchio, K., Nehman, S., Tracy, J.K. and Scalea, T.M. (2006) Impact of Obesity in the Critically Ill Trauma Patient: A Prospective Study. Journal of the American College of Surgeons, 203, 533-538. http://dx.doi.org/10.1016/j.jamcollsurg.2006.07.001

[5] McLaughlin, J.R. and Lee, K.R. (2006) The Outcome of Total Hip Replacement in Obese and Non-Obese Patients at 10- to 18-Year. Journal of Bone and Joint Surgery, 88, 1286-1292. http://dx.doi.org/10.1302/0301-620X.88B10.17660

[6] Upasani, V.V., Caltoum, C., Petcharaporn, M., et al. (2008) Does Obesity Affect Surgical Outcomes in Adolescent Idiopathic Scoliosis? Spine, 33, 295-300. http://dx.doi.org/10.1097/BRS.0b013e3181624573

[7] Erdil, M., Bilsel, K., Sungur, M., Dikmen, G., Tuncer, N., Polat, G., Elmadag, N.M., Tuncay, I. and Asik, M. (2013) Does Obesity Negatively Affect the Functional Results of Arthroscopic Partial Meniscectomy? A Retrospective Cohort Study. Arthroscopy, 29, 232-237. http://dx.doi.org/10.1016/j.arthro.2012.08.017

[8] Harrison, M.M., Morrell, J. and Hopman, W.M. (2004) Influence of Obesity on Outcome after Knee Arthroscopy. Arthroscopy, 20, 691-695. http://dx.doi.org/10.1016/S0749-8063(04)00589-4

[9] Fukubayashi, T. and Kurosawa, H. (1980) The Contact Area and Pressure Distribution Pattern of the Knee: A Study of Normal and Osteoarthritic Knee Joints. Acta Orthopaedica Scandinavica, 51, 871-879. http://dx.doi.org/10.3109/17453678008990887

[10] Jaiswal, P.K., Bentley, G., Carrington, R.W., Skinner, J.A. and Briggs, T.W. (2012) The Adverse Effect of Elevated Body Mass Index on Outcome after Autologous Chondrocyte Implantation. Journal of Bone and Joint Surgery, 94, 1377-1381. http://dx.doi.org/10.1302/0301-620X.94B10.29388

[11] Mardani-Kivi, M., Mirbolook, A., Karimi Mobarakeh, M., Khajeh Jahromi, S. and Hassanzadeh, R. (2015) Effect of Obesity on Arthroscopic Treatment of Anterolateral Impingement Syndrome of the Ankle. Journal of Foot and Ankle Surgery, 54, 13-16. http://dx.doi.org/10.1053/j.jfas.2014.09.005

[12] Collins, J.A., Beutel, B.G., Garofolo, G. and Youm, T. (2015) Correlation of Obesity with Patient-Reported Outcomes and Complications after Hip Arthroscopy. Arthroscopy, 31, 57-62. http://dx.doi.org/10.1016/j.arthro.2014.07.013

[13] Japour, C., Vohra, P., Giorgini, R. and Sobel, E. (1996) Ankle Arthroscopy: Follow-Up Study of 33 Ankles-Effect of Physical Therapy and Obesity. Journal of Foot and Ankle Surgery, 35, 199-209.

http://dx.doi.org/10.1016/S1067-2516(96)80097-4 